Gefässchirurgie 2020 $25: 587-602$ https://doi.org/10.1007/s00772-020-00688-3 Angenommen: 9. August 2020 Online publiziert: 18 . September 2020 ๑) Der/die Autor(en) 2020

C.-A. Behrendt ${ }^{1} \cdot$ U. Rother ${ }^{2}$ - G. Rümenapf ${ }^{3} \cdot$ C. Uhl ${ }^{4}$ - D. Böckler $\cdot$ H. Görtz ${ }^{4}$ • F. Heidemann ${ }^{1} \cdot$ Kommission PAVK und Diabetisches Fußsyndrom der DGG e.V.

'Forschungsgruppe GermanVasc, Klinik und Poliklinik für Gefäßmedizin, Universitäres Herz- und Gefäßzentrum UKE Hamburg, Universitätsklinikum Hamburg-Eppendorf, Hamburg, Deutschland ${ }^{2}$ Gefäßchirurgische Abteilung, Universitätsklinikum Erlangen, Erlangen, Deutschland

${ }^{3}$ Oberrheinisches Gefäßzentrum Speyer, Diakonissen-Stiftungs-Krankenhaus Speyer, Speyer, Deutschland ${ }^{4}$ Klinik für Gefäßchirurgie, Bonifatius Hospital Lingen, Lingen, Deutschland

${ }^{5}$ Klinik für Gefäßchirurgie und Endovaskuläre Chirurgie, Universitätsklinikum Heidelberg, Heidelberg, Deutschland

\title{
Geschlechterspezifische Unterschiede bei der endovaskulären Behandlung der peripheren arteriellen Verschlusskrankheit - Eine systematische Literaturübersicht
}

Frauen scheinen gegenüber Männern andere oder sogar atypische Symptome ihrer Erkrankungen wahrzunehmen, so etwa bei der Herzinsuffizienz [2, 9], dem Vorhofflimmern [53] oder der koronaren Herzkrankheit [50]. Zahlreiche Studien konnten außerdem geschlechterspezifische Unterschiede bei den Behandlungsergebnissen relevanter Herz-KreislaufErkrankungen nachweisen [38, 54]. In den 10-Jahres-Ergebnissen der ACST-1Studie konnte ein geringer, aber signifikanter Behandlungsvorteil bei der offenchirurgischen Karotis-Thrombendarteriektomie bei Frauen nachgewiesen werden, was sich letztlich in der Versorgungsrealität niedergeschlagen hat [20]. Bei der komplexen endovaskulären Therapie von Aortenerkrankungen konnte das weibliche Geschlecht als unabhängiger Prädiktor für das Auftreten von Blutungskomplikationen [5, 49] sowie spinalen Ischämien [22, 49] nachgewiesen werden. Hier gibt es bisher allerdings noch keine allgemein akzeptierten geschlechterspezifischen Empfehlungen.

Die periphere arterielle Verschlusskrankheit (PAVK) zählt mit mehr als 200 Mio. Betroffenen weltweit (davon ca. 40 Mio. in Europa) [16, 29] als Volkskrankheit und damit zu den wichtigsten Manifestationen der Atherosklerose. Obwohl Frauen in retrospektiven Beobachtungsstudien etwa 40 bis $50 \%$ dieser Zielpopulation ausmachen, ist ihr Anteil in randomisierten Studien mit nur $20 \%$ gering [26].

Die besondere Komplexität der zahlreichen miteinander interagierenden Risikofaktoren und der verfügbaren Behandlungsalternativen erschweren Forschungsvorhaben zu dieser Zielpopulation, unterstreichen allerdings auch den Stellenwert von geschlechterspezifischen Studien. So ist die Prävalenz von Komorbiditäten, wie etwa Diabetes, Bluthochdruck oder Dyslipidämie eindeutig auch vom Geschlecht abhängig [3, 52]. Zentrale Risikofaktoren, wie Nikotinkonsum, unterliegen dabei einem zeitlichen Wandel mit bereits messbaren Auswirkungen auf die geschlechterspezifische Krebsprävalenz. In einer kürzlich veröffentlichten Studie zur optimalen Arzneimittelverordnung der symptomatischen PAVK konnte ein signifikanter Nachteil für Frauen bei der Verordnung von Statinen nachgewiesen werden, was 


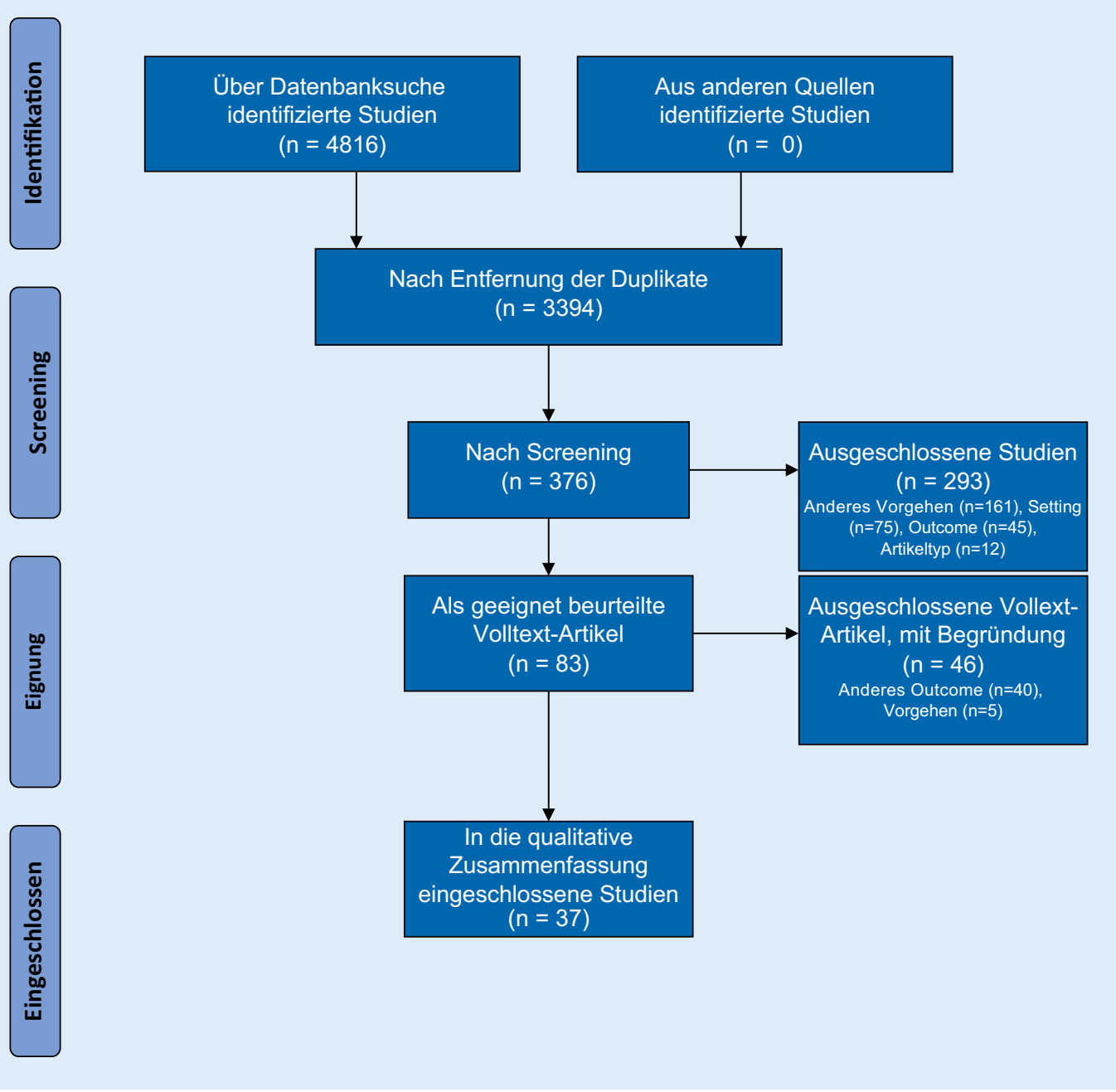

Abb. $1<$ Flowchart

auf eine Präferenz der behandelnden Ärzte und eine unterschiedliche Compliance beider Geschlechter hindeutet [44]. Es zeigen sich auch relevante Unterschiede bei der Inanspruchnahme der Rehabilitation, bei der Mobilität und in den sozialen Fürsorgestrukturen zwischen Männern und Frauen.

Trotz dieser Indizien für die Existenz geschlechterspezifischer Unterschiede beinhalten die aktuell gültigen Leitlinien bisher keine Anmerkungen bzw. klare Empfehlungen zu einer geschlechtersensitiven Diagnostik oder Behandlung der PAVK [1, 10, 19, 32].

Dieser Artikel gibt einen umfassenden Überblick über die verfügbare Literatur zu geschlechterspezifischen Unterschieden bei der endovaskulären Behandlung der symptomatischen PAVK.

\section{Methoden}

Die Berichterstattung dieser systematischen Literatursuche entspricht den Empfehlungen des Preferred Reporting Items for Systematic Reviews and MetaAnalyses (PRISMA)-Statements [35].

\section{Einschlusskriterien}

Für diesen Artikel wurde eine systematische Literatursuche durchgeführt. Prospektive und retrospektive Beobachtungsstudien (z. B. Register-, Datenbankoder Routinedatenstudien) zur invasiven endovaskulären Revaskularisation der symptomatischen PAVK wurden begutachtet, sofern sie über die Endpunkte Sterblichkeit oder Amputation getrennt für beide Geschlechter berichteten.

Ausgeschlossen wurden Übersichtsartikel, Metaanalysen, Leitlinien oder
Konsensusdokumente sowie interventionelle klinische Studien.

\section{Patient, Intervention, Comparison, Outcome (PICO)-Schema}

\section{Types of participants}

Männer und Frauen jeden Alters mit symptomatischer PAVK (Claudicatio intermittens, Fontaine-Stadium II vs. kritische Extremitätenischämie, FontaineStadien III-IV).

\section{Types of interventions}

Perkutane endovaskuläre Interventionen zur Revaskularisation chronischer Gefäßstenosierungen oder -verschlüsse der unteren Extremitäten. 
Gefässchirurgie 2020 $25: 587-602$ https://doi.org/10.1007/s00772-020-00688-3

(c) Der/die Autor(en) 2020

\section{C.-A. Behrendt · U. Rother · G. Rümenapf · C. Uhl · D. Böckler · H. Görtz · F. Heidemann · Kommission PAVK und Diabetisches Fußsyndrom der DGG e.V. \\ Geschlechterspezifische Unterschiede bei der endovaskulären Behandlung der peripheren arteriellen Verschlusskrankheit - Eine systematische Literaturübersicht}

\section{Zusammenfassung}

Hintergrund. Es gibt Hinweise auf geschlechterspezifische Unterschiede bei der Entwicklung, Diagnostik und Behandlung der symptomatischen peripheren arteriellen Verschlusskrankheit (PAVK). Obwohl Frauen in der Literatur etwa $50 \%$ der behandelten Kohorten ausmachen, ist deren Anteil in kontrollierten randomisierten Studien zur PAVK gering. Leitlinien enthalten bisher kaum geschlechtersensitive Empfehlungen, und die Ergebnisse retrospektiver Beobachtungsstudien widersprechen sich. Diese systematische Literaturrecherche soll verfügbare Beobachtungsstudien zu Geschlechterunterschieden bei der endovaskulären Behandlung der symptomatischen PAVK identifizieren.

\begin{abstract}
Material und Methoden. Es wurde eine systematische Literaturanalyse auf der Basis einer PubMed-Recherche mit dem Schwerpunkt auf retrospektive Beobachtungsstudien zum Vergleich der Behandlungsergebnisse zwischen Männern und Frauen durchgeführt. Ergebnisse. Insgesamt konnten 37 Publikationen identifiziert werden, die eine Kohorte von 5.489.558 Patientinnen und Patienten (40,3\% Frauen) beschreiben. Fünf Studien nutzten Propensity Score Matching zur Kontrolle von Störfaktoren. In 23 Studien wurden Behandlungsergebnisse nach der Dauer des Krankenhausaufenthalts bzw. nach 30 Tagen berichtet. Während 10 Studien einen Behandlungsnachteil bei Frauen
\end{abstract}

identifizieren konnten, berichteten 6 Studien über einen Behandlungsnachteil bei Männern. Die weiteren 21 Studien konnten keine eindeutigen Unterschiede zeigen. Schlussfolgerungen. Die verfügbare Real-World-Evidenz lässt aufgrund der methodischen Limitationen der eingeschlossenen Studien und deren unzureichender Vergleichbarkeit keine eindeutige Aussage zu geschlechterspezifischen Unterschieden bei der endovaskulären Behandlung der PAVK zu.

Schlüsselwörter

Real-World-Evidence · Versorgungsforschung . Gender · Qualitätsindikatoren · Endovaskuläre Techniken

\section{Gender-specific disparities in endovascular treatment of peripheral arterial occlusive disease: a systematic review of the literature}

\begin{abstract}
Background. There is evidence for gender disparities in the development, diagnostics and treatment of symptomatic peripheral arterial occlusive disease (PAOD). Although women represent $50 \%$ of treated cohorts in the literature, they only make up a small proportion in randomized trials on PAOD. Up to now, guidelines have included hardly any gender-sensitive recommendations and the results of observational studies are contradictory. This systematic literature review is aimed at identifying available observational studies on gender differences in the endovascular treatment of symptomatic PAOD.
\end{abstract}

Materials and Methods. A systematic review of the literature was carried out using PubMed, focusing on retrospective observational studies comparing outcomes between men and women.

Results. A total of 37 articles were identified constituting a cohort of 5,489,558 patients of both genders ( $40.3 \%$ women). Propensity score matching was used in five studies to address confounders. Twenty-three studies reported outcomes beyond the index hospital stay and 30 days, respectively. While 10 studies were able to identify disadvantages in females, 6 studies reported disadvantages in men. A further 21 studies were not able to identify any clear disparities.

Conclusions. Owing to methodological limitations and the inadequate comparability of the studies included, the available realworld evidence does not allow any distinct conclusions regarding gender-specific disparities in the endovascular treatment of PAOD.

\section{Keywords}

Real world evidence Health care research . Gender · Quality indicator · Endovascular techniques

\section{Types of outcome measures}

Sterblichkeit und Amputation nach perkutaner endovaskulärer Revaskularisation getrennt nach Männern vs. Frauen.

\section{Suchstrategie}

Über die biomedizinische Datenbank MEDLINE (über PubMed, US National Library of Medicine) wurde nach Originalarbeiten gesucht, die über die Endpunkte Sterblichkeit oder Amputation nach perkutaner endovaskulärer
Revaskularisation der symptomatischen PAVK berichten.

Für die Suche wurde eine Kombination der englischsprachigen Suchbegriffe bzw. sinnvoller Synonyme [„PAVK“ oder „Claudicatio intermittens“ oder „kritische Extremitätenischämie“] und [„Outcome“ oder „Sterblichkeit“ oder „Amputation“] verwendet. Die Suche wurde im Januar 2020 und im April 2020 durchgeführt. Alle Publikationen mit Veröffentlichungsdatum bis 30. April 2020 wurden berücksichtigt.

\section{Studienselektion}

Die identifizierten Studien wurden unabhängig von zwei Reviewern begutachtet (Franziska Heidemann, Christian-Alexander Behrendt). Aus den eingeschlossenen Studien wurden die nachfolgenden Informationen extrahiert: Autor und Publikationsjahr, Anzahl Patient*innen oder Prozeduren, Verteilung der Geschlechter, genutzte Datenbasis, Land der Erhebung, Zeitraum der Rekrutierung, Art und Umfang der statistischen 


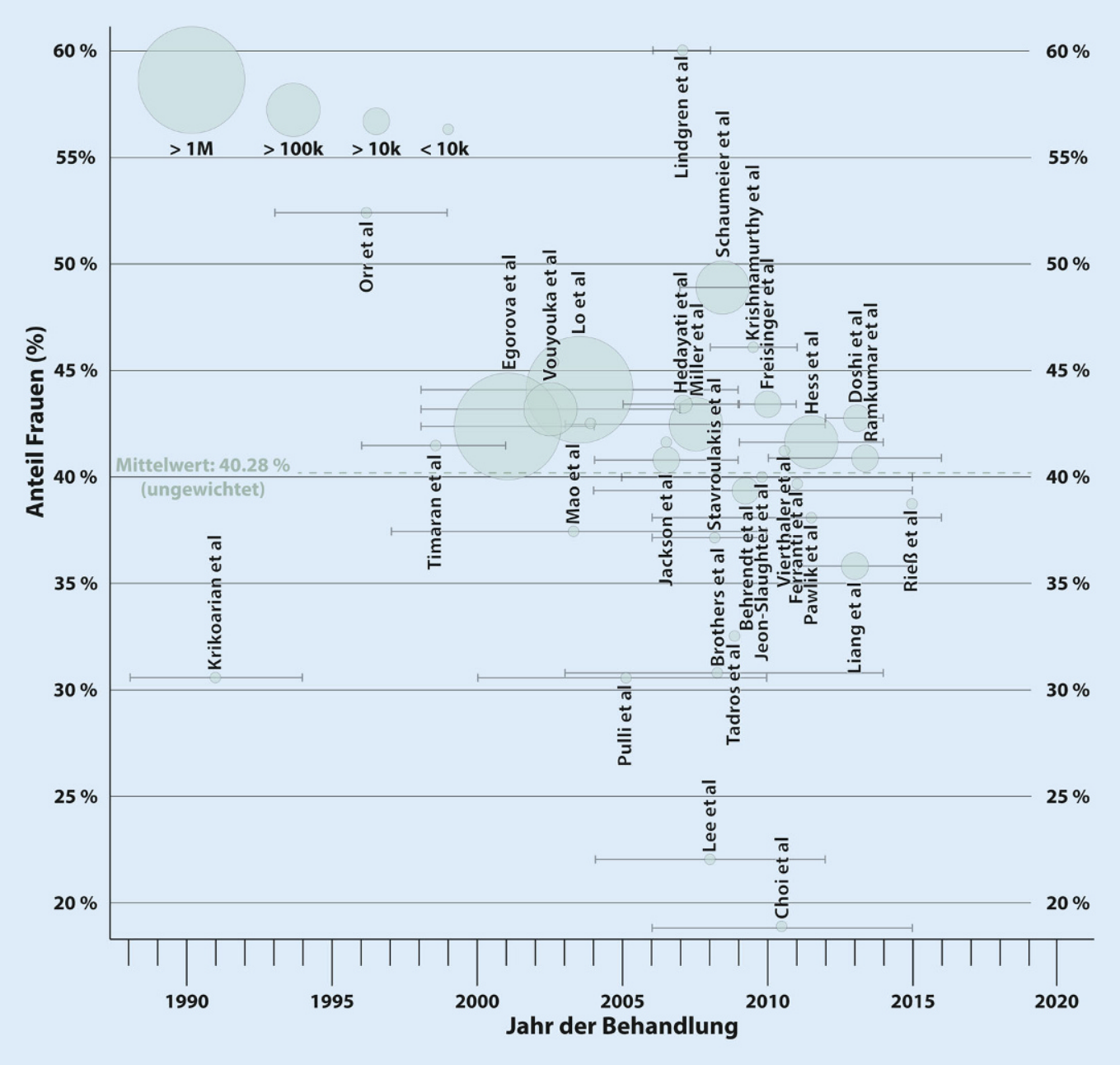

Abb. $2<$ Anzahl eingeschlossener Patient*innen und Anteil an Frauen der Beobachtungsstudien seit 1988
Analysen, Behandlungsverfahren, Indikation, primäre Endpunkte.

\section{Ergebnisse}

Durch den Suchalgorithmus konnten insgesamt 4816 Studien in PubMed identifiziert werden. Nach der Entfernung von 1422 Duplikaten verblieben 3394 Artikel, die auf Titel- und Abstractebene analysiert wurden. Hiervon sind insgesamt 376 Artikel in die Volltextanalyse eingegangen (• Abb. 1).

\section{Beschreibung der Studienpopula- tionen}

Unter den 37 in die qualitative Synthese eingeschlossenen Publikationen (insgesamt 5.489.558 Patient*innen, 1988 bis 2016) variierte der Anteil an Frauen zwischen $17,9 \%$ und $60,0 \%$ in den Studienpopulationen $(42,28 \%$ im ungewichteten
Mittel) (•Abb. 2). Die Anzahl an eingeschlossenen Patient ${ }^{*}$ innen variierte zwischen $n=74$ und $n=2.400 .000$.

Während vor 2010 überwiegend monozentrische Studien publiziert wurden, besteht die Datenbasis zwischen 2010 und 2016 größtenteils aus multizentrischen Studien.

Die Follow-up-Dauer variierte deutlich. In 14 Studien wurden lediglich perioperative Ergebnisse bis zu einer Dauer von 30 Tagen berichtet. In 23 Studien wurden mittel- und langfristige Behandlungsunterschiede bis zu 5 Jahren berichtet.

Eine Kontrolle von Störfaktoren durch Propensity Score Matching erfolgte in 5 Studien, während 32 Studien adjustierte Regressionsanalysen auswiesen.

\section{Geschlechterunterschiede}

Beim Vergleich der geschlechterspezifischen Behandlungsergebnisse wurden unterschiedliche Endpunkte berichtet (- Tab. 1). Bei 21 Studien wurden keine eindeutigen Geschlechterunterschiede nachgewiesen. Während 10 Studien (darunter 1 mit Propensity Score Matching) über einen Nachteil für Frauen berichteten $[7,8,14,27,36,37,40,41,46$, 60], gaben 6 Studien (darunter $1 \mathrm{mit}$ Propensity Score Matching) einen Behandlungsnachteil für Männer an [6, 17, 21, 23, 31, 59].

Bei den Studien, die einen Behandlungsnachteil für Frauen berichteten, wurden eine höhere Krankenhaussterblichkeit $[8,14,37,41,60]$, höhere 1-Jahres-Reinterventionsraten [27], höhere 2-Jahres-Reinterventionsraten [46], höhere 2-Jahresraten bezüglich kardiovaskulärer Composite-Endpunkte $[7,8$, 
40], höhere 1-Jahres-Sterblichkeit [27, 36] und höhere 2-Jahres-Sterblichkeit [8, 40] bei Frauen vs. Männern angegeben.

Bei den Studien, die einen Behandlungsnachteil für Männer berichteten, wurden eine höhere Krankenhaussterblichkeit und Amputationsrate [17], höhere 1-Jahresraten bezüglich kardiovaskulärer Composite-Endpunkte [21, 23], höhere 1-Jahres-Reinterventionsraten [21] und höhere 1-Jahresraten bezüglich amputationsfreiem Überleben [31, 59] bei Männern vs. Frauen angegeben.

\section{Diskussion}

Die vorliegende systematische Literaturrecherche konnte insgesamt 37 Beobachtungsstudien identifizieren, die über geschlechterspezifische Behandlungsergebnisse nach endovaskulärer Revaskularisation der symptomatischen PAVK berichtet haben. Insgesamt zeigte sich dabei eine deutliche Varianz bei den Kohorten, Einschlusskriterien, Analysemethoden und Endpunkten.

Während 21 Studien keine eindeutigen geschlechterspezifischen Unterschiedefeststellen konnten, berichteten 10 Studien über einen Behandlungsnachteil bei Frauen und 6 Studien über einen Behandlungsnachteil bei Männern.

Diese uneindeutige Evidenzbasis, die sich aus sehr inhomogenen Real-WorldDatenquellen generiert, mag ursächlich dafür sein, dass bis heute keine klaren geschlechterspezifischen Empfehlungen in den Leitlinien zur Diagnostik und Behandlung der PAVK enthalten sind [1, 19]. Dabei unterstreicht die zunehmende Anzahl an Publikationen über einen Zeitraum von mehr als 20 Jahren, dass diese Fragestellung für die Forschung weiterhin interessant und klinisch relevant ist. Obwohl Frauen fast die Hälfte der behandelten Kohorten ausmachen, ist ihr Anteil in prospektiven randomisierten Studien weiterhin ausgesprochen gering [26]. Es gibt zudem eindeutige Hinweise auf eine Unterversorgung mit leitliniengerechten Arzneimitteln bei Frauen, was einen Einfluss auf langfristige Behandlungsergebnisse nahelegt und die Hypothese begründet [44].

Miller et al. haben fast 140.000 Patienten ( $43 \%$ Frauen) aus dem Nationwide
Inpatient Sample zwischen 2003 und 2012 analysiert [41]. Eingegangen sind dort sowohl endovaskuläre als auch offen-chirurgische Revaskularisationen der Claudicatio intermittens und kritischen Extremitätenischämie. Hinsichtlich der Krankenhaussterblichkeit zeigte sich ein geringer, aber statistisch signifikanter Unterschied $(1,0 \%$ bei Frauen vs. $0,8 \%$ bei Männern, $p<0,05)$. In den multivariaten Analysen zeigte sich weibliches Geschlecht als unabhängiger Risikofaktor für die Sterblichkeit in der Gruppe der Claudicatio intermittens. Die große Fallzahl hat vermutlich einen Einfluss auf die Signifikanz trotz geringer Gruppenunterschiede. Die kurze Followup-Dauer und Wahl des Endpunktes wecken allerdings Zweifel an der klinischen Relevanz. Eine längere Follow-up-Dauer von bis zu 2 Jahren (376 Tage im Median) anhand des multizentrischen Registers der Vascular Quality Initiative (VQI) konnten Ramkumar et al. analysieren [46]. Etwa 58.000 Patienten (41\% Frauen) mit endovaskulären Behandlungen der femoro-poplitealen Strombahn zwischen 2010 und 2016 wurden analysiert. Hinsichtlich der technischen Endpunkte reinterventionsfreies Überleben und verschlussfreies Überleben nach 2 Jahren zeigten sich schlechtere Ergebnisse bei Frauen. In einer weiteren großen Analyse von Daten der multizentrischen United States Premier Healthcare Database konnten etwa 381.000 Patienten (42\% Frauen) hinsichtlich geschlechterspezifischer Unterschiede bei 1-Jahresraten an Major adverse limb events (MALE) untersucht werden [23]. In der multivariaten Analyse zeigte sich ein unabhängiger Behandlungsnachteil für Männer. In diese Analyse gingen allerdings nur etwa $80 \%$ endovaskuläre Verfahren ein und die Indikationen schlossen auch die akute Extremitätenischämie ein, was die Vergleichbarkeit deutlich einschränkt. Lo et al. konnten etwa 1,8 Mio. Patienten (44\% Frauen) mit Behandlungen zwischen 1998 und 2009 im Nationwide Inpatient Sample identifizieren und auswerten [37]. Die Nachteile bei der Krankenhaussterblichkeit bei Frauen bestätigen weitestgehend die anderen Studien und unterliegen den gleichen Limitationen. Interessant an dieser Auswertung ist allerdings, dass etwa 70.000 Prozeduren $(3,7 \%)$ als Reinterventionen bei zuvor behandelten Patienten durchgeführt wurden. Diese Beobachtung schränkt die Nutzung von fallbasierten Datensätzen ohne longitudinale Verknüpfung substanziell ein, da eine Verzerrung nicht auszuschließen ist. Dies gilt insbesondere bei Studien zur Behandlung der PAVK, was auch eine umfassende Analyse von Routinedaten der BARMER zwischen 2008 und 2016 ergeben hat [29]. Etwa $23 \%$ der mehr als 200.000 Behandlungsfälle wurden als wiederholte Behandlung durchgeführt.

Zusammenfassend schränken die methodischen Limitationen der verfügbaren Studien eine klare Bewertung der uneindeutigen Datenbasis ein. Die in dieser Arbeit enthaltenen Studien konnten zwar weitere Hypothesen generieren, bleiben die valide Beantwortung der Fragestellung allerdings schuldig. Es bleibt zu erwähnen, dass die Forschung zur PAVK generell durch sehr inhomogene Patientenkollektive und uneinheitlichen Behandlungsregime (z.B. Klassifikation, Verfahren, gerinnungswirksame Medikation, Nachsorge) betroffen ist. Dieser Umstand ist angesichts der großen Bedeutung dieser Zielerkrankung besonders bemerkenswert.

Retrospektive Beobachtungsstudien sollten die Ein- und Ausschlusskriterien klar und eng definieren und zwischen grundsätzlich unterschiedlichen Gruppen nach Möglichkeit stratifizieren (z. B. ,intermittent claudication“ [IC] vs. "chronic limb-threatening ischaemia“ [CLTI]). Die sehr unterschiedlichen Endpunkte erschweren zudem die Vergleichbarkeit und unterstreichen die Nutzung allgemein konsentierter Qualitätsindikatoren der PAVK-Behandlung. Hierzu liegen mehrere Konsensusempfehlungen vor, die interdisziplinär durchgeführt wurden [48]. 


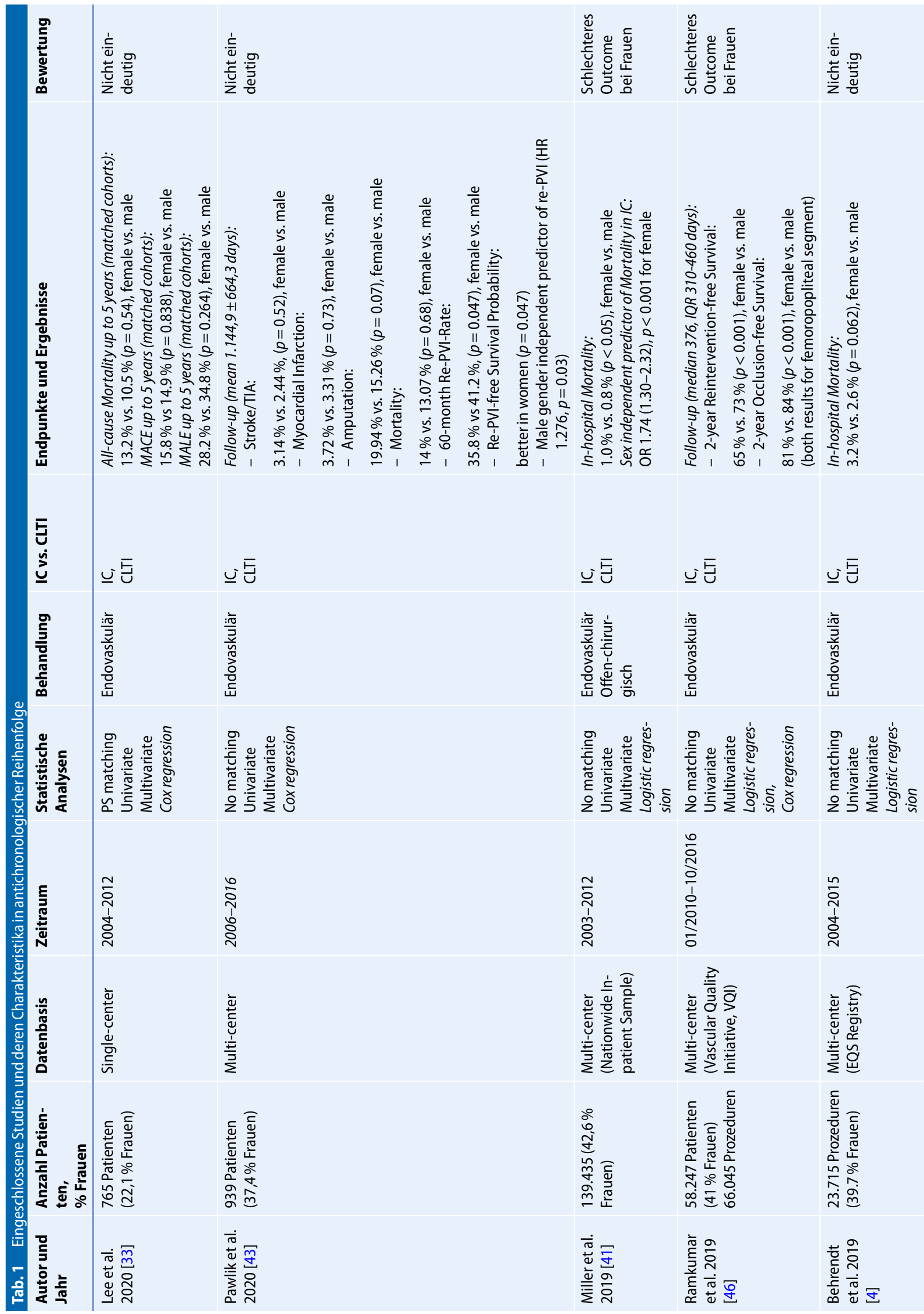




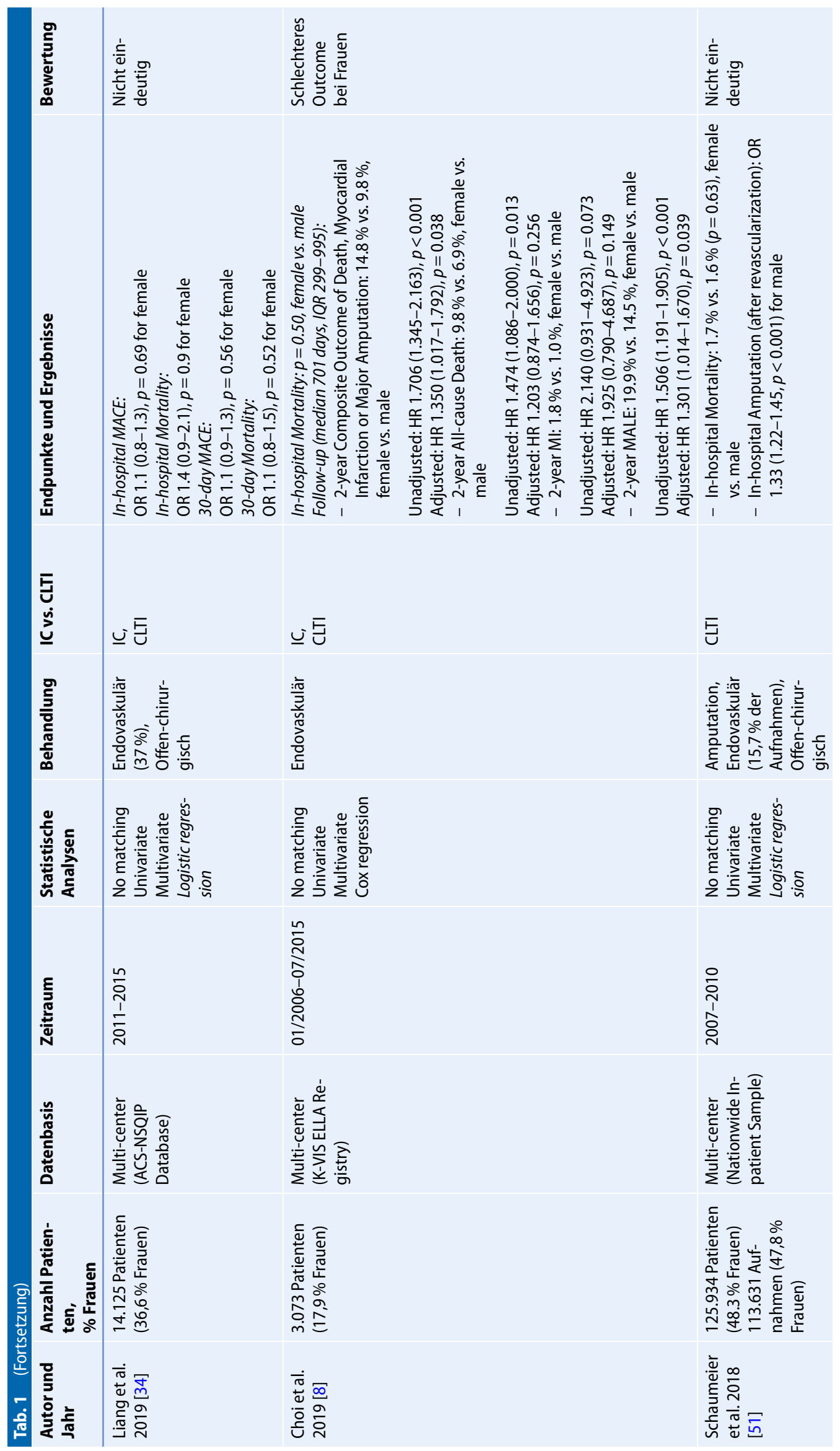




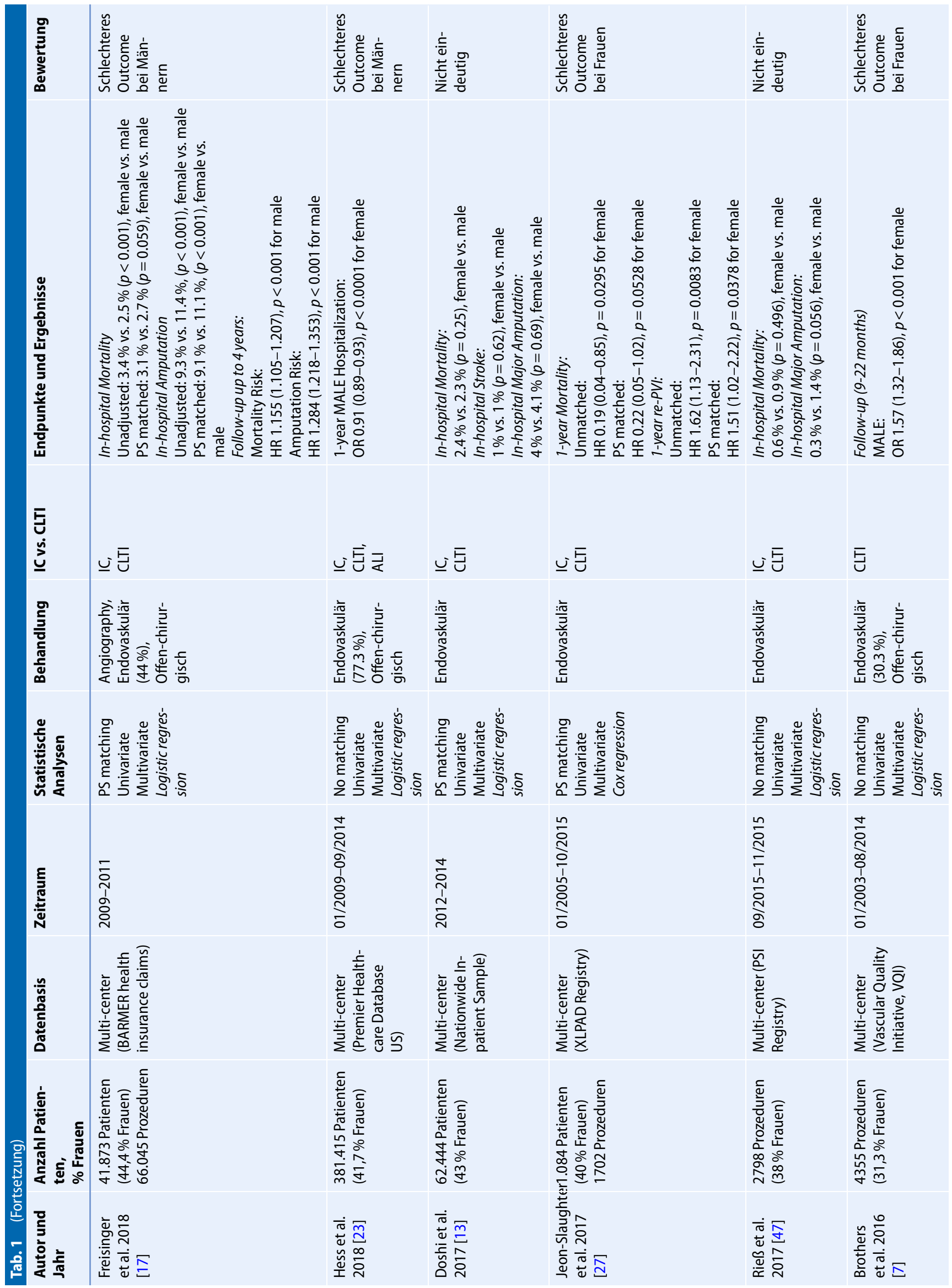




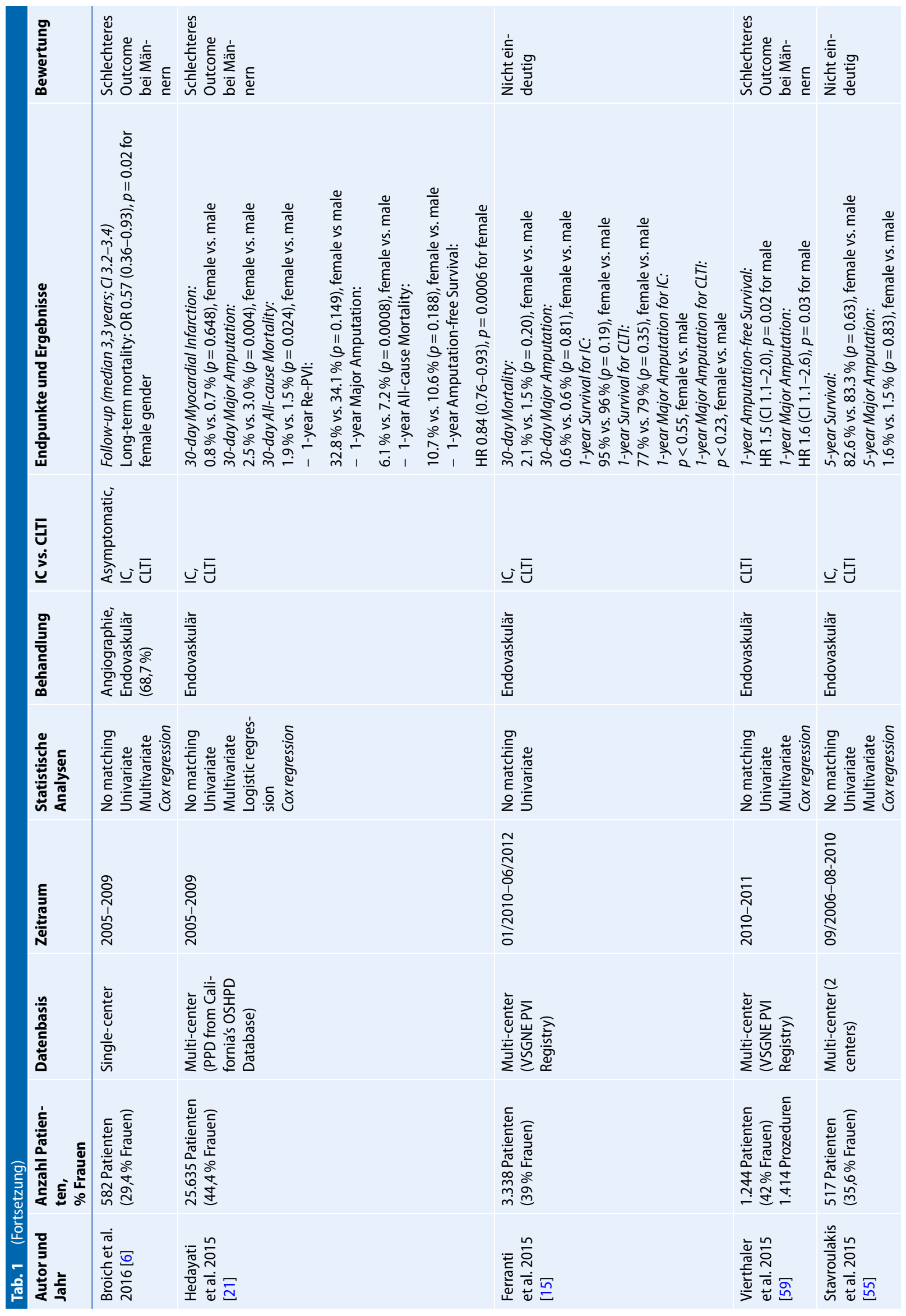




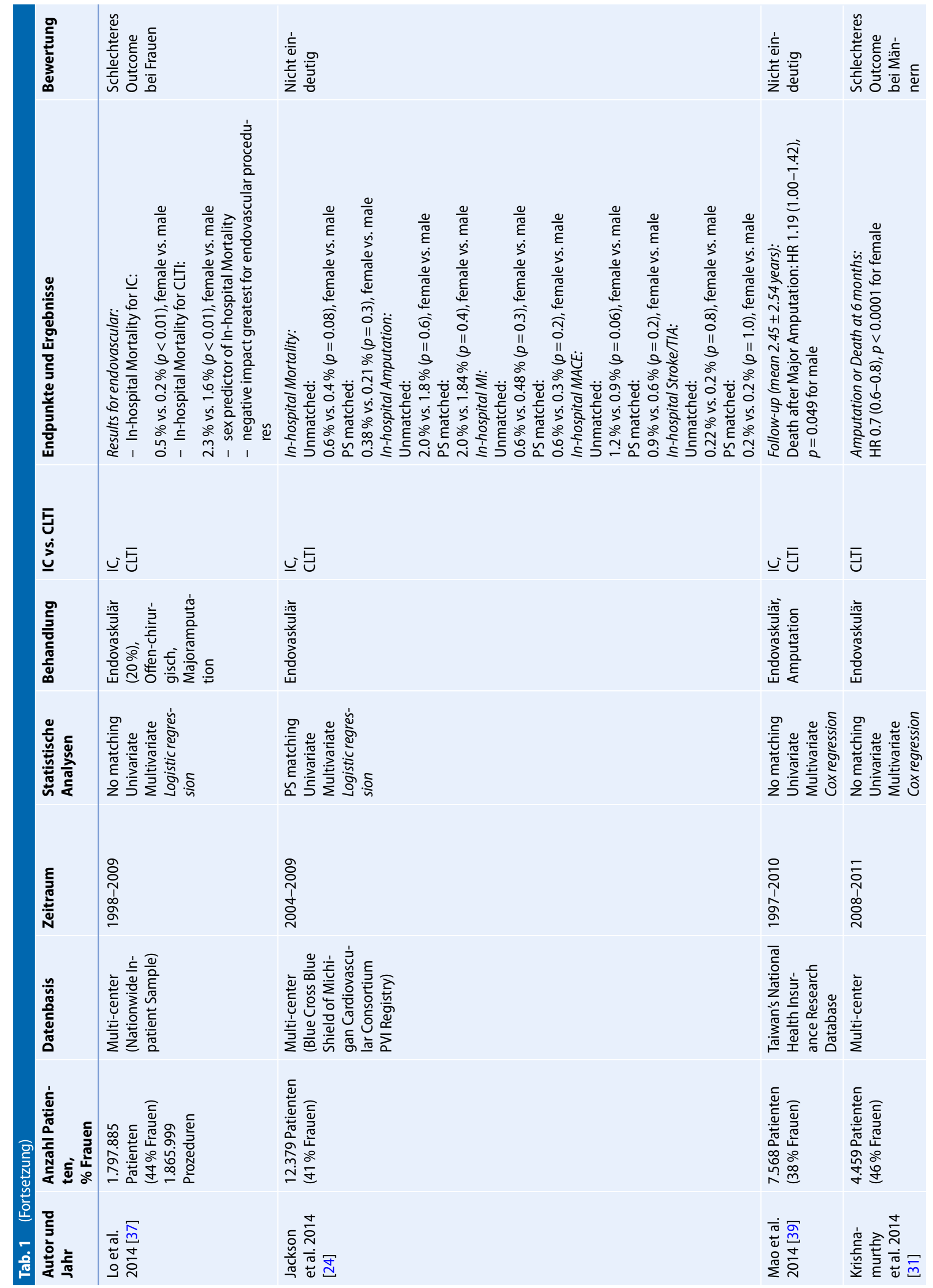




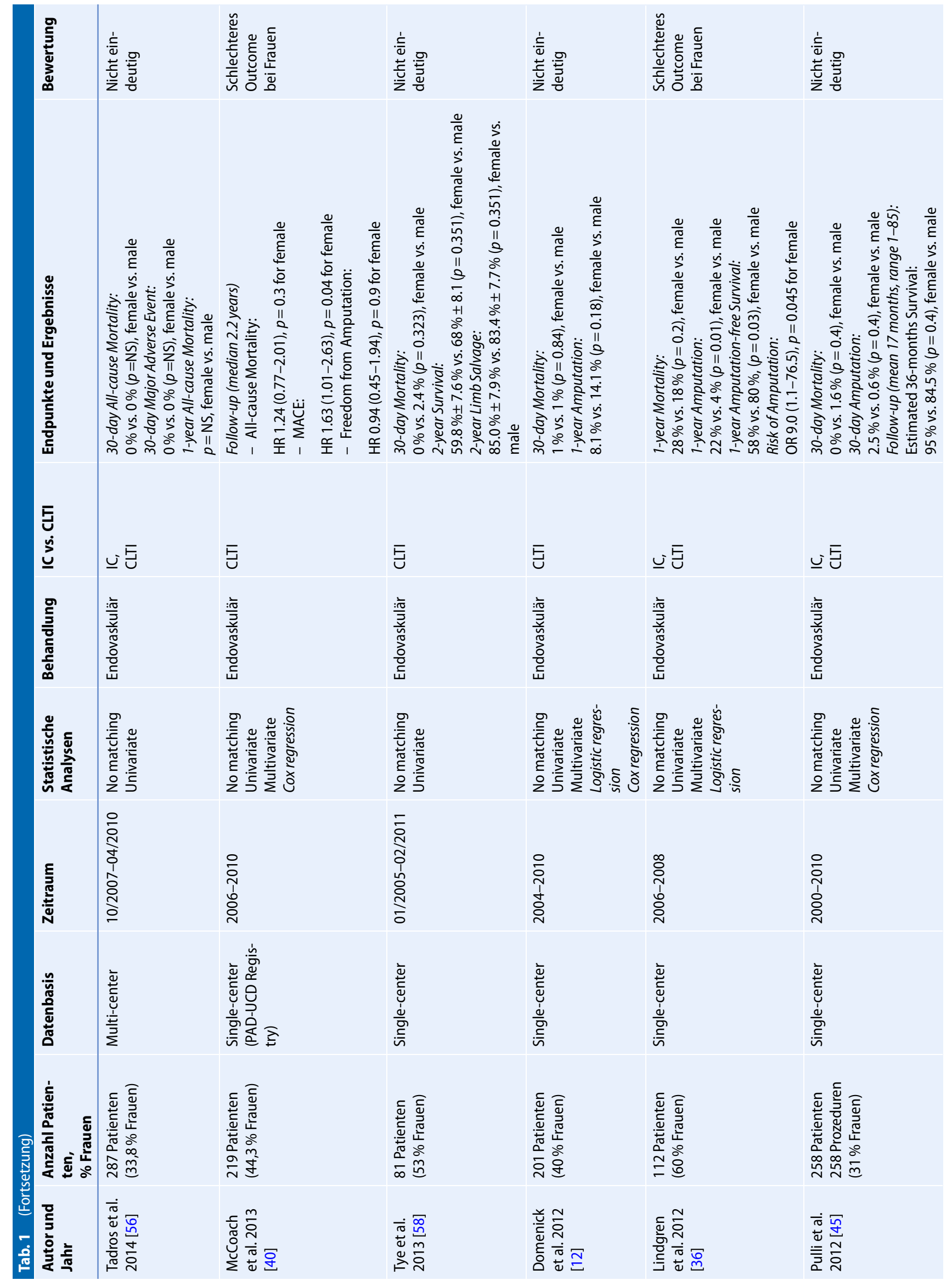




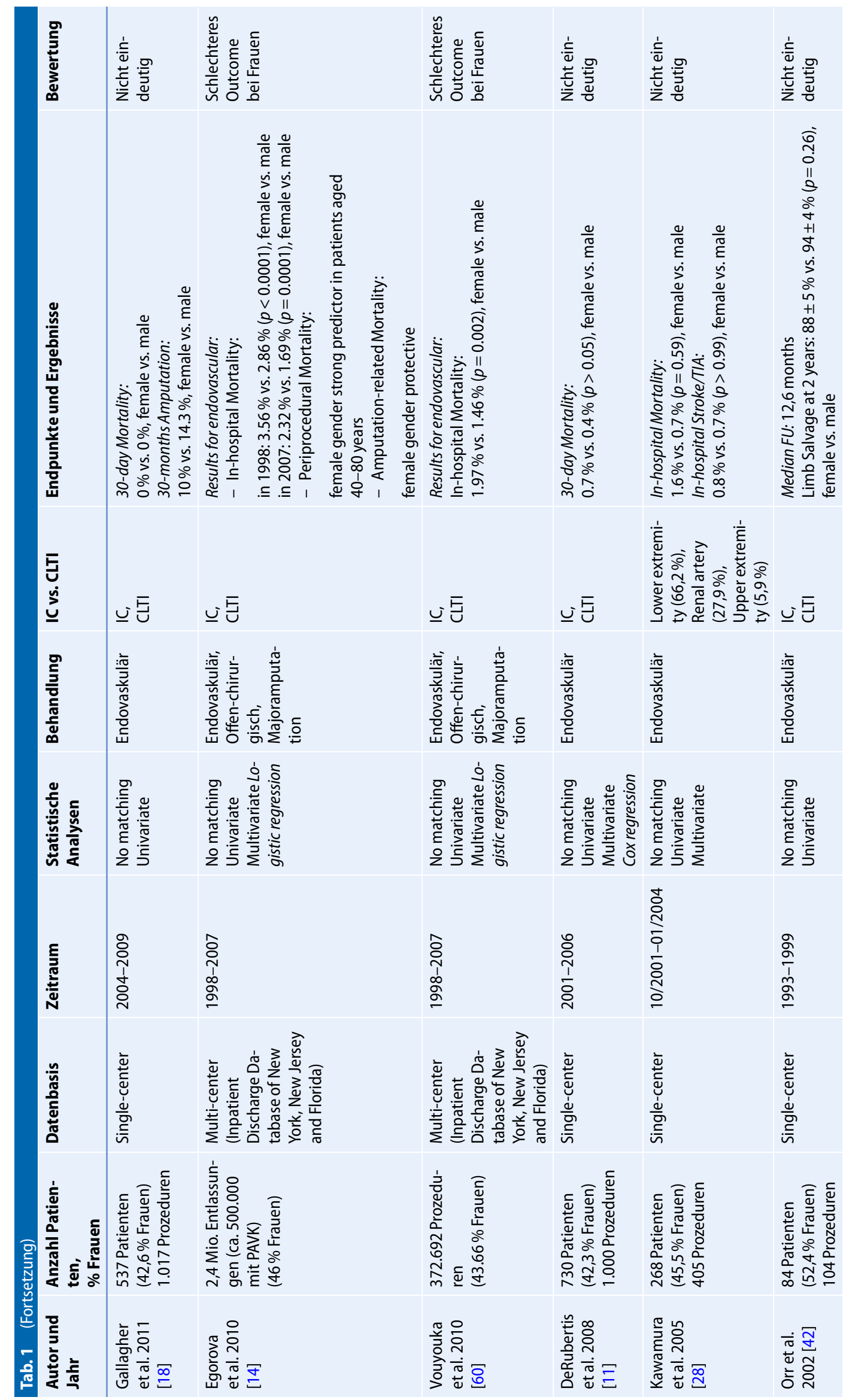




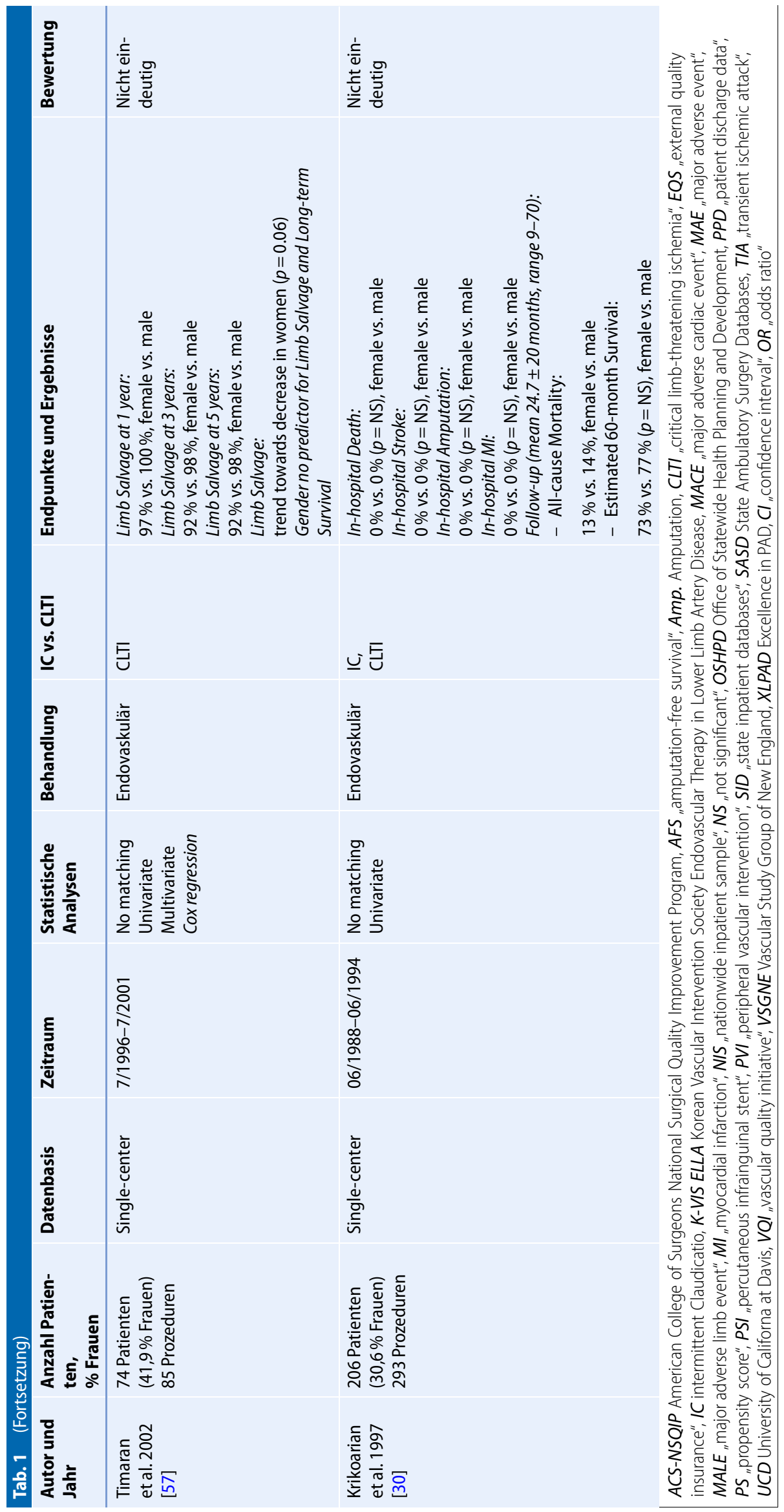




\section{Fazit für die Praxis}

- Obwohl Frauen im Mittel etwa $\mathbf{4 0 \%}$ der behandelten Kohorten ausmachten, ist deren Anteil in prospektiven kontrollierten Studien weiterhin niedrig.

- Insgesamt ist die Evidenzbasis zu geschlechterspezifischen Unterschieden nach perkutaner endovaskulärer Behandlung der symptomatischen peripheren arteriellen Verschlusskrankheit (PAVK) weiterhin uneindeutig. Es gibt sowohl Studien, die über einen Nachteil für Frauen als auch für Männer bzw. keine eindeutigen Unterschiede berichtet haben.

- Die wenigsten der verfügbaren Studien haben eine adäquate Kontrolle wesentlicher Confounder, z. B. mittels Matching-Verfahren, vorgenommen.

- Das GenderReality-Projekt der Forschungsgruppe GermanVasc verfolgt das Ziel, geschlechterspezifische Unterschiede in der Diagnostik und Behandlung von zentralen HerzKreislauf-Erkrankungen in Deutschland zu untersuchen.

- Mit der IDOMENEO- und RABATTStudie stehen multimethodale und mehrstufige Konsortialprojekte zur Verfügung, die sich mit der Qualitätsentwicklung in der invasiven Behandlung der peripheren arteriellen Verschlusskrankheit beschäftigen.

\section{Korrespondenzadresse}

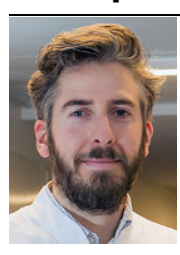

Dr. C.-A. Behrendt

Forschungsgruppe

GermanVasc, Klinik und Poliklinik für Gefäßmedizin, Universitäres Herz- und Gefäßzentrum UKE Hamburg, Universitätsklinikum Hamburg-Eppendorf Martinistr. 52, 20246 Hamburg, Deutschland behrendt@hamburg.de

Funding. Open Access funding provided by Projekt DEAL.

\section{Einhaltung ethischer Richtlinien}

Interessenkonflikt. C.-A. Behrendt, U. Rother, G. Rümenapf, C. Uhl, D. Böckler, H. Görtz und F. Heidemann geben an, dass kein Interessenkonflikt besteht.

Für diesen Beitrag wurden von den Autoren keine Studien an Menschen oder Tieren durchgeführt. Für die aufgeführten Studien gelten die jeweils dort angegebenen ethischen Richtlinien.

Open Access. Dieser Artikel wird unter der Creative Commons Namensnennung 4.0 International Lizenz veröffentlicht, welche die Nutzung, Vervielfältigung, Bearbeitung, Verbreitung und Wiedergabe in jeglichem Medium und Format erlaubt, sofern Sie den/die ursprünglichen Autor(en) und die Quelle ordnungsgemäß nennen, einen Link zur Creative Commons Lizenz beifügen und angeben, ob Änderungen vorgenommen wurden.

Die in diesem Artikel enthaltenen Bilder und sonstiges Drittmaterial unterliegen ebenfalls der genannten Creative Commons Lizenz, sofern sich aus der Abbildungslegende nichts anderes ergibt. Sofern das betreffende Material nicht unter der genannten Creative Commons Lizenz steht und die betreffende Handlung nicht nach gesetzlichen Vorschriften erlaubt ist, ist für die oben aufgeführten Weiterverwendungen des $\mathrm{Ma}$ terials die Einwilligung des jeweiligen Rechteinhabers einzuholen.

Weitere Details zur Lizenz entnehmen Sie bitte der Lizenzinformation auf http://creativecommons.org/ licenses/by/4.0/deed.de.

\section{Literatur}

1. Aboyans V, Ricco JB, Bartelink MEL et al (2018) Editor's choice-2017 ESC guidelines on the diagnosis and treatment of peripheral arterial diseases, in collaboration with the European Society for Vascular Surgery (ESVS). Eur J Vasc Endovasc Surg 55(3):305-368. https://doi.org/10. 1016/j.ejvs.2017.07.018

2. Agvall B, Dahlstrom U (2001) Patients in primary health care diagnosed and treated as heart failure, with special reference to gender differences. Scand JPrim Health Care 19:14-19

3. Anonymous (2017) 2. Classification and diagnosis of diabetes: standards of medical care in diabetes-2018. Diabetes Care 41:S13-S27

4. Behrendt CA, Bischoff MS, Schwaneberg T et al (2019) Population based analysis of gender disparities in 23,715 percutaneous endovascular revascularisations in the metropolitan area of Hamburg. Eur JVasc Endovasc Surg 57:658-665

5. Behrendt CA, Debus ES, Schwaneberg $T$ et al (2020) Predictors of bleeding or anemia requiring transfusion in complex endovascular aortic repair and its impact on outcomes in health insurance claims. JVasc Surg 71:382-389

6. Broich EM, Reinecke H, Malyar NM et al (2016) Long-term mortality after invasive diagnostic and endovascular revascularization in PAD patients. Int Angiol 35:516-525

7. Brothers TE, Zhang J, Mauldin PD et al (2016) Predicting outcomes for infrapopliteal limbthreatening ischemia using the Society for Vascular Surgery Vascular Quality Initiative. J Vasc Surg 63:114-124 (e115)
8. Choi KH, Park TK, Kim J et al (2019) Sex differences in outcomes following endovascular treatment for symptomatic peripheral artery disease: an analysis from the K-VIS ELLA registry. J Am Heart Assoc 8:e10849

9. Cleland JG, Swedberg K, Follath F et al (2003) The EuroHeart Failure survey programme-a survey on the quality of care among patients with heart failure in Europe. Part 1: patient characteristics and diagnosis. Eur Heart J 24:442-463

10. Conte MS, Bradbury AW, Kolh P et al (2019) Global vascular guidelines on the management of chronic limb-threatening ischemia. Eur J Vasc Endovasc Surg 58:S1-S109 (e133)

11. Derubertis BG, Vouyouka A, Rhee SJ et al (2008) Percutaneous intervention for infrainguinal occlusive disease in women: equivalent outcomes despite increased severity of disease compared with men. J Vasc Surg 48:150-157 (discussion 157-158)

12. Domenick N, Saqib NU, Marone LK et al (2012) Impact of gender and age on outcomes of tibial artery endovascular interventions in critical limb ischemia. Ann Vasc Surg 26:937-945

13. Doshi R, Shah P, Meraj P (2017) Gender disparities among patients with peripheral arterial disease treated via endovascular approach: a propensity scorematched analysis. J Interv Cardiol 30:604-611

14. Egorova N, Vouyouka AG, Quin J et al (2010) Analysis of gender-related differences in lower extremity peripheral arterial disease. J Vasc Surg 51:372-378 (e371; discussion 378-379)

15. Ferranti KM, Osler TM, Duffy RP et al (2015) Association between gender and outcomes of lower extremity peripheral vascular interventions. JVasc Surg 62:990-997

16. Fowkes FG, Rudan D, Rudan I et al (2013) Comparison of global estimates of prevalence and risk factors for peripheral artery disease in 2000 and 2010: a systematic review and analysis. Lance 382:1329-1340

17. Freisinger $\mathrm{E}$, Malyar NM, Reinecke $\mathrm{H}$ et al (2018) Low rate of revascularization procedures and poor prognosis particularly in male patients with peripheral artery disease-a propensity score matched analysis. Int J Cardiol 255:188-194

18. Gallagher KA, Meltzer AJ, Ravin RA et al (2011) Gender differences in outcomes of endovascular treatment of infrainguinal peripheral artery disease. Vasc Endovascular Surg 45:703-711

19. Gerhard-Herman MD, Gornik HL, Barrett $C$ et al (2017) 2016 AHA/ACC guideline on the management of patients with lower extremity peripheral artery disease: a report of the American College of Cardiology/American Heart Association Task Force on Clinical Practice Guidelines. J Am Coll Cardiol 69:e71-e126

20. Halliday A, Harrison M, Hayter E et al (2010) 10-year stroke prevention after successful carotid endarterectomy for asymptomatic stenosis (ACST-1): a multicentre randomised trial. Lancet 376:1074-1084

21. Hedayati N, Brunson A, Li CS et al (2015) Do women have worse amputation-free survival than men following endovascular procedures for peripheral arterial disease? An evaluation of the California state-wide database. Vasc Endovascular Surg 49:166-174

22. Heidemann F, Kolbel T, Kuchenbecker J et al (2020) Incidence, predictors, and outcomes of spinal cord ischemia in elective complex endovascular aortic repair: an analysis of health insurance claims. JVasc Surg 72:837-848 
23. Hess CN, Rogers RK, Wang TY et al (2018) Major adverse limb events and 1-year outcomes after peripheral artery revascularization. J Am Coll Cardiol 72:999-1011

24. Jackson EA, Munir K, Schreiber Tetal (2014) Impact of sex on morbidity and mortality rates after lower extremity interventions for peripheral arterial disease: observations from the Blue Cross Blue Shield of Michigan Cardiovascular Consortium. JAm Coll Cardiol 63:2525-2530

25. Jagodzinski A, Johansen C, Koch-Gromus U et al (2020) Rationale and design of the Hamburg City Health study. Eur JEpidemiol 35:169-181

26. Jelani QU, Petrov M, Martinez SC et al (2018) Peripheral arterial disease in women: an overview of risk factor profile, clinical features, and outcomes. Curr Atheroscler Rep 20:40

27. Jeon-Slaughter H, Tsai S, Kamath P et al (2017) Comparison of lower extremity endovascular intervention outcomes in women versus men. Am JCardiol 119:490-496

28. Kawamura A, Piemonte TC, Nesto RW et al (2005) Impact of gender on in-hospital outcomes following contemporary percutaneous intervention for peripheral arterial disease. J Invasive Cardiol 17:433-436

29. Kreutzburg T, Peters F, Riess HC et al (2020) Editor's choice-comorbidity patterns among patients with peripheral arterial occlusive disease in Germany: a trend analysis of health insurance claims data. Eur JVasc Endovasc Surg 59:59-66

30. Krikorian RK, Kramer PH, Vacek JL (1997) Percutaneous revascularization of lower extremity arterial disease in females compared to males. J Invasive Cardiol 9:333-338

31. Krishnamurthy V, Munir K, Rectenwald JE et al (2014) Contemporary outcomes with percutaneous vascular interventions for peripheral critical limb ischemia in those with and without polyvascular disease. Vasc Med 19:491-499

32. Lawall H, Huppert P, Espinola-Klein C et al (2017) German guideline on the diagnosis and treatment of peripheral artery disease - a comprehensive update 2016. Vasa 46:79-86

33. LeeMS, ChoiBG, Hollowed Jetal (2020) Assessment of sex differences in 5-year clinical outcomes following endovascular revascularization for peripheral artery disease. Cardiovasc Revasc Med 21:110-115

34. Liang P, Li C, O'donnell TFX et al (2019) Inhospital versus postdischarge major adverse events within 30 days following lower extremity revascularization. JVasc Surg 69:482-489

35. Liberati A, Altman DG, Tetzlaff J et al (2009) The PRISMA statement for reporting systematic reviews and meta-analyses of studies that evaluate health care interventions: explanation and elaboration. JClin Epidemiol 62:e1-e34

36. Lindgren $\mathrm{H}$, Gottsater $\mathrm{A}$, Hermansson $\mathrm{K}$ et al (2012) Gender differences in outcome after stent treatment of lesions in the femoropopliteal segment. Scand J Surg 101:177-183

37. LoRC,Bensley RP, Dahlberg SEetal (2014) Presentation, treatment, and outcome differences between men and women undergoing revascularization or amputation for lower extremity peripheral arterial disease. J Vasc Surg 59:409-418 (e403)

38. Magnussen C, Niiranen TJ, Ojeda FM et al (2017) Sex differences and similarities in atrial fibrillation epidemiology, risk factors, and mortality in community cohorts: results from the BiomarCaRE Consortium (Biomarker for Cardiovascular Risk Assessment in Europe). Circulation 136:1588-1597
39. Mao CT, Tsai ML, Wang CY et al (2014) Outcomes and characteristics of patients undergoing percutaneous angioplasty followed by belowknee or above-knee amputation for peripheral artery disease. Plos One 9:e111130

40. Mccoach CE, Armstrong EJ, Singh $S$ et al (2013) Gender-related variation in the clinical presentation and outcomes of critical limb ischemia. Vasc Med 18:19-26

41. Miller SM, Sumpio BJ, Miller MS et al (2019) Higher inpatient mortality for women after intervention for lifestyle limiting claudication. Ann Vasc Surg 58:54-62

42. Orr JD, Leeper NJ, Funaki B et al (2002) Gender does not influence outcomes after iliac angioplasty. Ann Vasc Surg 16:55-60

43. Pawlik A, Januszek R, Ruzsa Z et al (2020) Gender differences and long-term clinical outcomes in patients with chronic total occlusions of infrainguinal lower limb arteries treated from retrograde access with peripheral vascular interventions. Adv Med Sci 65:197-201

44. Peters F, Kreutzburg T, Riess HC etal (2020) Optimal pharmacological treatment of symptomatic peripheral arterial occlusive disease and evidence of female patient disadvantage: an analysis of health insurance claims data. Eur J Vasc Endovasc Surg.https://doi.org/10.1016/j.ejvs.2020.05.001

45. Pulli R, Dorigo W, Pratesi G et al (2012) Genderrelated outcomes in the endovascular treatment of infrainguinal arterial obstructive disease. J Vasc Surg 55:105-112

46. Ramkumar N, Suckow BD, Brown JR et al (2019) Role of sex in determining treatment type for patients undergoing endovascular lower extremity revascularization. J Am Heart Assoc 8:e13088

47. Riess HC, Debus ES, Heidemann F et al (2017) Gender differences in endovascular treatment of infrainguinal peripheral artery disease. Vasa 46:296-303

48. Riess HC, Debus ES, Schwaneberg T et al (2018) Indicators of outcome quality in peripheral arterial disease revascularisations-a Delphi expert consensus. Vasa 47:491-497

49. Riess HC, Debus ES, Schwaneberg T et al (2019) Gender disparities in fenestrated and branched endovascular aortic repair. Eur J Cardiothorac Surg 55:338-344

50. Schannwell CM, Schoebel FC, Lazica D et al (2000) Differences in the clinical performance and initial diagnosis in women with suspected coronary artery disease. Dtsch Med Wochenschr 125:1417-1423

51. Schaumeier MJ, Hawkins AT, Hevelone ND et al (2018) Association of treatment for critical limb ischemia with gender and hospital volume. Am Surg 84:1069-1078

52. Scheerbaum M, Langenbach C, Scheerbaum Pet a (2017) Prevalence of cardiovascular risk factors among 28,000 employees. Vasa 46:203-210

53. Schnabel RB, Pecen L, Ojeda FM et al (2017) Gender differences in clinical presentation and 1-year outcomes in atrial fibrillation. Heart 103:1024-1030

54. Sorensen NA, Neumann JT, Ojeda F et al (2018) Relations of sex to diagnosis and outcomes in acute coronary syndrome. J Am Heart Assoc. https://doi. org/10.1161/JAHA.117.007297

55. Stavroulakis K, Donas KP, Torsello G et al (2015) Gender-related long-term outcome of primary femoropopliteal stent placement for peripheral artery disease. JEndovasc Ther 22:31-37
56. Tadros RO, Faries PL, Rocha-Singh KJ et al (2014) The impact of sex on angioplasty and primary stenting for femoropopliteal occlusive disease: results of the DURABILITY II trial. Ann Vasc Surg 28:1-9

57. Timaran $\mathrm{CH}$, Stevens $\mathrm{SL}$, Freeman $\mathrm{MB}$ et al (2002) Predictors for adverse outcome after iliac angioplasty and stenting for limb-threatening ischemia. JVasc Surg 36:507-513

58. Tye A, Han DK, Tadros RO et al (2013) Percutaneous intervention for infrageniculate arterial disease in women may be associated with better outcomes when compared to men. JVasc Surg 57:706-713

59. Vierthaler L, Callas PW, Goodney PP et al (2015) Determinants of survival and major amputation after peripheral endovascular intervention for critical limb ischemia. J Vasc Surg 62:655-664 (e658)

60. Vouyouka AG, Egorova NN, Salloum A et al (2010) Lessons learned from the analysis of gender effect on risk factors and procedural outcomes of lower extremity arterial disease. J Vasc Surg 52:1196-1202 
Hier steht eine Anzeige.

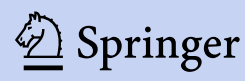

